\title{
Review Article \\ Biomarkers Discovery for Colorectal Cancer: A Review on Tumor Endothelial Markers as Perspective Candidates
}

\author{
Lukasz Pietrzyk ${ }^{1,2}$ \\ ${ }^{1}$ Department of Didactics and Medical Simulation, Chair of Human Anatomy, Medical University of Lublin, Lublin, Poland \\ ${ }^{2}$ Department of General, Oncological and Minimally Invasive Surgery, 1st Military Clinical Hospital, Lublin, Poland \\ Correspondence should be addressed to Lukasz Pietrzyk; lukasz.pietrzyk@wp.pl
}

Received 3 July 2016; Revised 2 October 2016; Accepted 16 October 2016

Academic Editor: Gad Rennert

Copyright (c) 2016 Łukasz Pietrzyk. This is an open access article distributed under the Creative Commons Attribution License, which permits unrestricted use, distribution, and reproduction in any medium, provided the original work is properly cited.

\begin{abstract}
Colorectal cancer (CRC) is the third most common cancer in the world. The early detection of CRC, during the promotion/progression stages, is an enormous challenge for a successful outcome and remains a fundamental problem in clinical approach. Despite the continuous advancement in diagnostic and therapeutic methods, there is a need for discovery of sensitive and specific, noninvasive biomarkers. Tumor endothelial markers (TEMs) are associated with tumor-specific angiogenesis and are potentially useful to discriminate between tumor and normal endothelium. The most promising TEMs for oncogenic signaling in CRC appeared to be the TEM1, TEM5, TEM7, and TEM8. Overexpression of TEMs especially TEM1, TEM7, and TEM8 in colorectal tumor tissue compared to healthy tissue suggests their role in tumor blood vessels formation. Thus TEMs appear to be perspective candidates for early detection, monitoring, and treatment of CRC patients. This review provides an update on recent data on tumor endothelial markers and their possible use as biomarkers for screening, diagnosis, and therapy of colorectal cancer patients.
\end{abstract}

\section{Introduction}

Colorectal cancer (CRC) is the third most common cancer in the world with approximately 1.4 million new cases diagnosed in 2012. The disease is a leading cause of death in approximately $50 \%$ of CRC patients [1]. The predictions are that the morbidity and mortality rates of CRC will increase due to unprecedented global trends in population aging and profound adverse effects of many lifestyle-related factors [2]. Overall 5-year survival for cancer limited to the colon is $95 \%$ and $82 \%$ for stages I and stage II, respectively. However, it decreases considerably to $61 \%$ for patients with regional spread to the lymph nodes (stage III) and only $8 \%$ for patients with distant metastases (stage IV) [3]. The early detection of CRC, before the promotion/progression stages, is an enormous challenge for a successful outcome and remains a fundamental problem in clinical approach [4]. Despite the continuous advancement in diagnostic and therapeutic methods (i.e., colonoscopy, flexible sigmoidoscopy, and stoolbased tests), effective in the reduction of mortality, the figures published by the National Cancer Institute indicate that still a significant number of individuals are diagnosed in later stages of CRC development (stages III and IV) [5].

Therefore, recent studies have been focusing on identification of sensitive and specific, noninvasive biomarkers that could detect the presence of CRC before it reaches advanced stages [6].

Recently a considerable attention is given to tumor endothelial cells (TECs). Abnormalities between tumor and normal endothelial cells open an opportunity to identify specific markers (tumor endothelial markers (TEMs)) linked to tumor angiogenesis. Markers that could distinguish physiological and pathological angiogenesis are an important issue for cancer detection $[7,8]$. It is widely accepted that biomarkers offer chances to establish prognostic indicator in CRC and for their perspective use in clinical applications. Inhibiting angiogenesis is an important strategy for current therapies of cancer patients [9]. Selective delivery of blocking molecules to tumor endothelium has become a major goal of current antiangiogenic treatment strategies for cancer. An ideal marker for such selective targeting would be highly expressed in tumor endothelium but absent or exceedingly 
rare in all nontumor endothelium. To date, few, if any, markers have been identified that meet such strict criteria.

This review provides an update on recent data on tumor endothelial markers (TEMs) and their possible use as biomarkers for screening, diagnosis, and therapy of colorectal cancer patients.

\section{Cancer Angiogenesis}

There are two distinct stages of tumor development: (i) the avascular growth phase and (ii) the vascular growth phase. In the first phase, the tumor is solid (size $<1-2 \mathrm{~mm}$, multicellular spheroids) and is dormant, noninvasive (carcinoma in situ). In the second phase, the spread of cancer may appear. Numerous studies have shown that avascular tumors are restricted in their growth potential due to the lack of a blood supply needed for cell-to-cell diffusion of nutrients and metabolites exchange. It is accepted that the solid tumor growth (size $>1-2 \mathrm{~mm}$ ) and its invasion are angiogenesisdependent [10].

The tumor may persist dormant for years after the primary neoplasm has been developed [11]. A significant turnoff point for the cancer growth, progression, and metastatic spread of cancer cells is angiogenesis [12]. The initiation of local small blood vessels development induces rapid, logarithmic tumor growth that follows tumor cells blood supply. The angiogenesis is a complex (multistage) process of new blood capillaries formation. The key stages include (i) initiation by endothelial cell activation (ECs), (ii) degradation of basement membrane, extracellular matrix, and pericyte detachment, (iii) proliferation and ingratiation of endothelial cells into the surrounding matrix, (iv) extensive enlargement of new vessels and stabilization of network (anastomosis), and (v) blood flow $[12,13]$.

The tumor blood vessels may have different origins. They may originate from preexisting neighboring blood vessels (termed "sprouting" angiogenesis) and from bone marrow-derived endothelial cell (EC) precursors (termed vasculogenesis) [14]. The mechanisms by which tumors promote new blood vessel formation are a complicated process that involves a complex and dynamic interaction between endothelial cells (ECs) and the corresponding extracellular and intracellular environment [15]. However, the molecular and cellular mechanisms that regulate angiogenesis have not been entirely explained. Many hypotheses have been proposed to clarify the tumor survival, the tumor growth, and its metastasizing to distant organs $[12,16]$. For example, basement membrane degradation and pericytes detachment are provoked by upsetting of the local cathepsin-cysteine protease inhibitor balance [17]. Rapid enlargement of vessel's size is related to, at least in part, the transport of macromolecules across venules in the vascular matrix. The vesiculovacuolar organelles are involved in the process $[18,19]$. The tumor blood vessels are dramatically different from normal blood vessels. These abnormalities involve morphological and functional disturbances is shown as follows [16, 20-24].

Morphological and functional characteristics of tumor blood vessels are as follows.

\section{Morphology}

It has disorganized structure without branchial pattern of arteries, veins, and capillaries.

It does not form monolayer of endothelial cells.

It has abnormal basement membrane of loose association with endothelial cells and varying thicknesses of type IV collagen layers.

Pericytes with free association with endothelial cells have cytoplasmic processes extending deep into the tumor tissue.

\section{Function}

It might collapse and impedes blood flow.

It does not have normal barrier function.

It is characterized by increased leakiness.

The most widely accepted model for the tumor growth is an angiogenic model. It suggests the tumor "switch on" and the tumor growth dependent on the balance of endogenous proangiogenic and antiangiogenic factors [25]. The mechanisms that lead to angiogenic phenotype activation (tumor "switch on") are associated with the expression of proangiogenic genes activated by production, secretion, and accumulation of proangiogenic factors that promote increased angiogenesis.

Therefore, one of the primary challenges for CRC early diagnosis is to identify a biomarker or a group of biomarkers that corresponds to early cancer stage of angiogenesis. Although angiogenic CRC-specific blood biomarkers have not been identified so far, several growth factors and their specific receptors have been recognized to induce and regulate angiogenesis in colorectal cancer. The most frequently described proangiogenic tumor "switch on" factors include vascular endothelial growth factors (VEGFs), fibroblast growth factor (FGF), platelet-derived growth factor (PDGF), transforming growth factors (TGFs), epidermal growth factor (EGF), angiopoietins (Angs), and others [6, 26]. Many in vitro models have shown that these molecules supported tumor angiogenesis and revealed that the degree of tumor angiogenesis is often closely linked to the level of these stimulating factors' concentration [11, 15, 17-19, 25, 26].

\section{Tumor Endothelial Cells (TECs)}

Histological abnormalities in blood vessels refer to the endothelium, pericytes, and basement membrane. Tumor endothelial cells (TECs) overexpress specific genes, such as tumor endothelial markers (TEMs) and epidermal growth factor receptors (EGFRs). The signaling system involves molecules that impact on pericyte/endothelial cell homeostasis $[27,28]$. Abnormal expression patterns of biomolecules (proteins, glycoprotein, and glycans) are well known to be specific for tumor endothelium. The endothelial cells change their properties depending on their origin, age, and the expression of cell surface antigens. Circulating endothelial 
cells (CECs) are derived from multiple sources, including bone marrow (circulating endothelial progenitors (CEPs)) and established vasculature (mature CECs). As a result of respective complements of cell surface molecules and receptors, the functions of endothelial cells are diverse [17].

\section{Endothelial Progenitor Cells (EPCs)}

A growing body of evidence indicates the endothelial progenitor cells (EPCs) are particularly important for tumor angiogenesis determining tumor growth and metastasis [29, 30]. EPCs have been first described in detail by Asahara et al. [31]. EPCs hierarchy is defined based on their behavior, proliferative potential, and vasculogenic ability [32]. In human, main populations of EPCs include early EPCs and putative adult EPCs, circulating or resident cells. However, they can achieve at least 100 population doublings (PDs).

EPCs express a variety of cell surface markers similar to those expressed by vascular endothelial cells, adhere to endothelium at sites of hypoxia/ischemia, and participate in new vessels formation [33]. Early EPCs (localized predominately in the bone marrow) are positive for CD133 (termed AC133, human prominin-1 surface antigen initially), CD34 antigen, and VEGFR-2 (vascular endothelial growth factor receptor-2). More mature EPCs (circulating in blood) express phenotype CD133(-)/CD34(+)/VEGFR-2(+). Mature EPCs display a phenotype VEGFR-2(+)/VE-cadherin(+)/von Willebrand factor $(+)(\mathrm{vWF})$ [34]. Furthermore, circulating in blood EPCs coexpress various molecules (platelet endothelial cell adhesion molecule-1 (PECAM termed as CD31)), CD146 immunoglobulin, and VE-cadherin (vascular endothelial) with different intensity $[31,34,35]$. These changes in antigens expression in endothelial progenitor cells suggest that at least two types of EPCs are present in the blood simultaneously and indicate that EPCs can change their properties in the blood [35]. It is speculated that EPCs potentials for proliferation and transformation into more mature EPCs are important in regulating the angiogenesis [36, 37]. However, it is still unclear at which point residual EPCs are induced to change into circulating EPCs, the endothelial-like subtype with angiogenic properties.

Furthermore, most of the endothelial markers used in assessing angiogenesis are not specific enough and are expressed in both normal and tumor tissues. However, a significant contribution to the issue has been made by Duda et al., who succeed in distinguishing between circulating endothelial cells (CECs) and leukocytes by using CD146 immunoglobulin in the blood of cancer patients [38].

\section{Tumor Endothelial Markers (TEMs)}

Due to the technical difficulties and problems with analyses of gene expression isolated from tumor endothelial cells, the studies on the tumor angiogenesis were carried out using normal endothelial cells (e.g., human umbilical vein endothelial cells) for a long time. The first who compared the gene expression in normal endothelial cells (ECs) and tumor endothelial cells (TECs) derivative from malignant colorectal tissues were St Croix et al. [39]. The serial analysis of gene expression (SAGE) in endothelial cells derived from blood vessels of normal cells and malignant colorectal tissues revealed 46 transcripts specifically elevated in TECs [40]. Nine transcripts were hoped to be unique to TECs and therefore were named tumor endothelial markers (TEMs). TEMs are potentially useful to discriminate between tumor and normal endothelial cells as 20-fold higher expression has been reported in human tumor endothelium [41]. It is speculated that tumor endothelial markers are likely to be most accessible to pharmacological agents, which is why TEMs are indicated as useful for pharmacological interventions in therapeutic targets [42].

Tumor endothelial markers (TEMs) belong to a family of proteins that are associated with tumor-specific angiogenesis $[7,8]$. Genes encoding TEMs display elevated expression during tumor angiogenesis and are conserved in mice and humans. Therefore, TEMs are potentially useful to sufficiently distinguished patients with cancer and noncancer as well as cancer patients with different tumor stages. However, some studies pointed out that some TEMs might be even overexpressed during physiological angiogenesis; for example, TEM2 and TEM6 and their expression are not restricted to tumor endothelial cells [40].

Studies investigating the expression of TEMs in human colorectal cancer (CRC) are limited. However, the most promising TEMs for oncogenic signaling in CRC appeared to be the TEM1, TEM5, TEM7, TEM7R, and TEM8. These tumor endothelial cells' markers display elevated expressions during tumor angiogenesis in CRC patients [8, 41, 43]. TEMs are transmembrane proteins containing putative domains that function as receptors; for example, TEM5 appears to be a seven-pass transmembrane receptor, whereas TEM1, TEM7, and TEM 8 pass the cell membrane once (Figure 1) $[44,45]$.

5.1. Tumor Endothelial Marker 1. Tumor endothelial marker 1 (TEM1, endosialin, and CD 248) is a $165 \mathrm{kDa}$ transmembrane glycoprotein of 757 amino acids. TEM1 consists of two domains: (i) a long-chain (670 amino acids) extracellular domain (ECD) and (ii) a short-chain (49 amino acids) cytoplasmic domain. The extracellular domain (ECD) of TEM1 consists of one C-type lectin-like, one Sushi, three EGFlike domain, and mucin-like stalk receptors. TEM1 has been localized on healthy and tumor endothelium and in stromal fibroblasts $[43,46]$. The expression of TEM1 is specifically higher in tumor endothelium (5-, 10-, and 20-fold) compared to the endothelium of normal tissues [39]. A significant increase of TEM1 expression in the stroma between distant or adjacent normal mucosa and primary tumor $(3 \%, 5 \%$, and $63 \%$, resp.) was documented in rectal cancer patients [47].

Two mechanisms are suggested to regulate TEM1 gene expression: (i) cell density and (ii) hypoxia [48, 49]. Opavsky et al. [48] observed the positive correlation of endogenous TEM1 expression with the density of NIH3T3 cells. Low level of TEM1 RNA was found in the sparse NIH3T3 cells. In contrast, the strongest induction of transcription was characteristic for NIH3T3 cells at full confluence. The upregulation of TEM1 expression is known to be induced by 


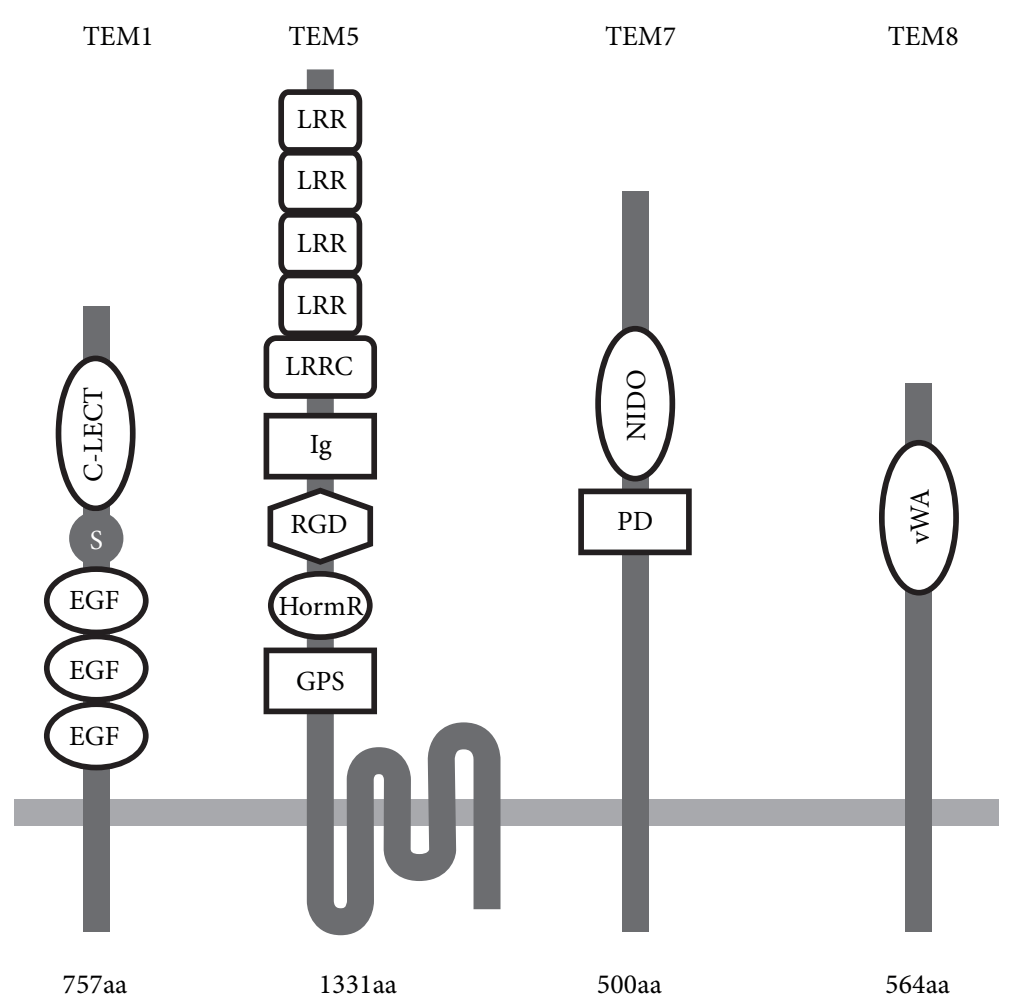

FIGURE 1: Predicted molecular structure of the tumor endothelial markers (TEM1, TEM5, TEM7, and TEM8). EGF: epidermal growth factorlike domain; S: Sushi domain; C-LECT: C-lectin domain; GPS: proteolysis site; HormR: hormone receptor domain; RGD: Arg-Gly-Asp motif; Ig: immunoglobulin-like domain; LRRC: leucine-rich repeat C-terminal domain; LRR: leucine-rich repeat; PD: plexin-like domain; NIDO: nidogen-like domain; vWA: von Willebrand type A domain; aa: amino acids chain length.

hypoxia, a condition well identified as a primary activator of angiogenesis in solid tumors [50]. Human cell lines (FIB3 placental fibroblasts and 42-MG-BA glioblastoma cells) exposed to hypoxia $\left(2 \% \mathrm{O}_{2}\right)$ were found to have significantly higher TEM1 gene expression compared to the same cell lines incubated in normoxia [49]. High TEM1 gene expression is partially mediated by an interaction between hypoxiainducible factor-2 (HIF-2) and the Ets-1 transcription factor. Subsequent studies have confirmed that HIF-2 can activate the TEM1 distal (enhancer) and proximal (core) promoter. The combination of indirect and direct promoter activation by HIF-2-Ets-1 is suggested. Direct TEM1 distal promoter activation involves the HIF-2 binding to a hypoxia-response element (HRE) site and the adjacent ETS-1-binding site. The indirect pathway engages binding the Ets- 1 and its two cognate EBS elements located in the proximal promoter of TEM1 [49, 51, 52].

TEM1 shows overexpression in tumor tissues. It was suggested that TEM1 is engaged in tumor invasion (progression and metastasis) all the more so because the absence of TEM1 expression reduced tumor growth [43]. Nanda et al. [53] using mouse xenograft model have shown an important functional role of TEM1 in growth and progression of abdominal tumors. The effect of Teml knockout (KO) on embryo development and vascularization in a wound healing assays (the wound sites morphology, number, and size of vessels at the site of the wounds) was not found. However, a considerable change in tumor growth pattern (alteration in vascularization, a decrease in bulk growth, prevention of the local invasion, and metastasis reduction) in HCT116 human colorectal cancer cells implanted into the serosal surface of the large intestines of Teml knockout (KO) mice has been documented. The lack of TEM1 gene expression resulted in the reduction of xenograft tumors aggressiveness; that is, smaller tumor volume and lower metastases rate (KO versus WT mice was $0 \%$ versus $33 \%$ of liver metastases) have been revealed [53].

TEM1 is elevated in a wide range of human carcinomas (breast, lung, pancreas, urinary bladder, brain glioma, and melanoma) [43, 54-58]. Rmali et al. [43] found overexpression of TEM1 in colon cancer tissues compared to normal tissues $(95.5 \%$ versus $38 \%$ of positive tissues for TEM1 expression, resp.; $p<0.01$ ). Zhang et al. [47] reported significantly lower expression of TEM1 in rectal cancer tissues when comparing TNM stage I with other stages: TNM II + III + IV (45\% versus $74 \%$, resp.; $p=0.03$ ). Accordingly, it was suggested that, in CRC patients, TEM1 expression correlates with the disease' advancement [43].

The elevated expression of TEM1 has also been found in pericytes (vascular cells that envelop the surface of the vascular tube) $[54,59]$. Pericytes are considered to be directly involved in regulation of blood vessels morphogenesis and play a critical role in cardiovascular homeostasis $[28,60]$. Evidently, modulation of pericyte function is likely to reduce 
normal and pathological angiogenesis. The absence of TEM1 expression in pericytes causes a decrease of larger and mature vessels and an increase of small and immature tumor vessels, indicating a considerable involvement of TEM1 in tumor angiogenesis and microvasculature maturation [53, 61]. Tomkowicz et al. described the molecular mechanism by which the tumor-stromal compartment can control tumor aggressiveness [54]. The suggested mechanism includes three phases: (i) an activation of TEM1 expression in perivascular cells and stromal fibroblasts by unknown factor, (ii) a modulation of tissue proteases resulting from interaction between TEM1 and extracellular matrix components that is pericytes or fibroblasts, and (iii) tumor vessels maturation and induction of cell-to-cell attachments and cells migration.

Since the expression of TEM1 in colorectal carcinogenesis has been documented, the TEM1 has become a potential diagnostic and therapeutic target molecule [39]. Preclinical murine model of ID8 tumors (a mouse ovarian surface epithelium cancer cell line) designed by Chacko et al. [62] showed the potential diagnostic utility of MORAb-004, a humanized $\operatorname{IgG1} / \kappa$ monoclonal antibody $(\mathrm{mAb})$ directed against human TEM1. In the immuno-PET examination, high specific and sensitive uptake of 125I-MORAb-004 and 124I-MORAb-004 in tumors compared to healthy tissue was observed (immunoreactivity approximately 90\%). Moreover, high tumor-to-background tissue contrast was achieved for 124I-MORAb-004. Similar immunoreactivity was demonstrated with 89Zr-MORAb-004 [63]. 89Zr-MORAb-004 was able to bind TEM1 and could distinguish between high and low TEM1 expression tissues, which has been evidenced in specific immuno-PET imaging of sarcoma cell lines xenografts [63].

St Croix et al. also pointed out the TEM1 was a promising prospect for use in cancer immunotherapy [39]. They showed that TEM1 was recognized by the monoclonal antibody called FB5, detectable immunohistochemically on blood vessels of a majority of human tumors. Moreover, Facciponte et al. [64] in murine colon cancer model (colon carcinoma line CT26) revealed significant tumor protection effect of TEM1TT vaccine (TEM1 cDNA fused to the minimal domain of the $\mathrm{C}$ fragment of tetanus toxoid). The TEM1-TT vaccine showed the power to break down TEM1 tolerance. TEM1TT vaccination exerted humoral antitumor activity through the significant increase of CD3 + $\mathrm{T}$ cells infiltration in the tumor (42 $\pm 5 \mathrm{CD} 3+$ cells/high-powered field [hpf] versus TT-vaccinated mice; $p<0.05)$. TEM1-TT-vaccinated tumors were characterized by significantly lower hemoglobin level and showed decreased area of perfusion and blood flux compared to TT-vaccinated tumors. The dependence suggests the functional disruption of tumor vasculature induced by TEM1-TT vaccine. Currently, clinical studies (phase I) are conducted to assess both biological and side effects of the MORAb-004 in patients with solid tumors $(n=$ $36)$, including colorectal cancer patients $(n=11)$ [65]. The MORAb-004 maximum tolerated dose at the level of $12 \mathrm{mg} / \mathrm{kg}$ has been defined, and treatment-emergence adverse events (i.e., fatigue $47.2 \%$, headache $36.1 \%$, pyrexia $22.2 \%$, chills $19.4 \%$, and nausea $13.9 \%$ ) have been noticed. Antitumor activity with stabilization of the disease at least for 106 days in patients with tumor of epithelial origin, including colorectal cancer patients, has been documented [65]. The mechanism of MORAb-004 action is not thoroughly revealed. However, the MORAb-004 mediated internalization and removing of TEM1 from the cells surface have been suggested as a possible one. The TEM1 involvement in the interaction with extracellular matrix proteins (i.e., fibronectin (FN), collagen types I and IV) and/or participation in signaling pathways (i.e., via the PDGF-receptor) are considered. Presumably, MORAb004 controls TEM1 adhesion to the extracellular matrix or regulates the TEM1 involvement in cell signaling pathways. In these ways, the MORAb-004 indirectly influences the tumorstromal cells communication. The phase II clinical studies have been designed and initiated in patients with colorectal cancer, melanoma, and soft tissue sarcoma to answer a role of MORAb-004 in adhesion, migration, survival, and proliferation of tumor [54, 65-67].

The TEM1 expression seems to be a promising target for cancer diagnosis and immunotherapy. The TEM1 expression could be detected by MORAb-004 in positron emission tomography. This allows classification and selections of patients with high TEM1 expression for more detailed diagnosis. Regarding that the MORAb-004 can inhibit endosialin/TEM1-mediated interaction with extracellular matrix, the MORAb-004-based immunotherapeutic product may block TEM1 and reduce carcinogenesis $[63,65]$.

5.2. Tumor Endothelial Marker 5. Tumor endothelial marker 5 (TEM5, G-protein-coupled receptor 124, and GPR124) is homologous to adhesion G-protein-coupled receptors (GPCRs) and belongs to a group of integral transmembrane proteins. TEM5 is composed of 1331 amino acids [68]. The protein bears long extracellular N-terminal part (N60), seven-pass domains, and an intracellular domain the $\mathrm{C}$ terminal part (C50). TEM5 extracellular domain [termed sTEM5 (soluble TEM5)] is composed of subdomains and motifs, that is, an immunoglobulin (Ig) domain, a hormone receptor (HormR), cryptic RGD (Arg-Gly-Asp) motif, a leucine-rich repeat (LRR) domain, a leucine-rich repeat Cterminal (LRRCT) domain, and a membrane proximal GPCR proteolysis site [69].

TEM5 activation is related to the cell-to-cell contacts during capillary morphogenesis [28]. TEM5 subdomains and motifs are involved in cell-cell and cell-matrix interactions. For example, RGD motif by binding the specific integrin (i.e., $\alpha v \beta_{3}$ ) receptors on the surface of endothelial cells modulates cell membrane functions, for example, adhesion, migration, and mediates endothelial cell survival during angiogenesis [70]. The C-terminal part (C50) domain interacts with the tumor suppressor protein human disc large (hDlg) [69]. It has been reported that the hDlg protein is involved in cell growth control and is known as a negative regulator of cell proliferation [71].

Interestingly, during capillary formation, TEM5 expression and upregulation can be induced by GTPase/Rac cascade [68]. It is currently clear that the cell proliferation in endothelial human umbilical vein endothelial cells (HUVEC) can be inhibited by blocking monoclonal TEM5 antibody. 
This creates the potential for the inhibition of cell divisions in endothelial tissues during capillary morphogenesis. Another study demonstrated a vital role of TEM5 in coactivation of the signaling pathway in endothelium brain development [72]. This fact together with TEM5 overexpression in tumor vasculature holds promise for the creation of new drugs that will block angiogenesis and cancer growth [41].

TEM5 architecture partly helps to explain multiple mechanisms of its action. The protein binds ligands (i.e., secretin, calcitonin) and activates many specific receptors located on the cell surface or inside the cell. In turn, TEM5 receptors are engaged in signal-transduction cascades and have the capacity for generation of mitogenic signals via currently only partly understood pathways. TEM5 is involved in the regulation of adenylate cyclases (AC) signaling system [68]. Consistent with this observation, TEM5 role in cell signaling pathways occurring during multiple cancer types development and progression is proposed. One of the presumable TEM5 actions is assistance in the interaction between the linked receptors and tyrosine kinase receptors, resulting in kinase inhibition [69]. Kinases overexpression enabled CRC progression and shortened cancer-specific survival [73]. These findings suggest that TEM5 is the important regulator in CRC spread [71].

TEM5 was highly expressed in endothelial cells and tumor stroma in human colon carcinoma xenograft compared to normal colonic tissue [41]. The expression of TEM5 is found during capillary morphogenesis or capillary network formation $[27,28]$. However, evidence for the TEM5 role in tumor angiogenesis has not been explained. Current report suggests TEM5 plays a key role in VEGF-induced tumor angiogenesis. Mouse xenograft tumor angiogenic vessels formation and in turn tumor growth were inhibited by silencing TEM5 in human endothelial cells. TEM5 regulated VEGF-induced tumor angiogenic processes in vitro including cell-cell interaction, permeability, migration, invasion, and tube formation [74]. In vitro study showed that knockdown of TEM5 in non-small cell lung cancer (NSCLC) reduces resistance to gefitinib [75]. This result indicates that TEM5 may contribute to the pathogenic angiogenesis. Hence, it appears to be a potential therapeutic target.

5.3. Tumor Endothelial Marker 7. Tumor endothelial marker 7 (TEM7, plexin domain containing 1, and PLXDC1) belongs to a group of transmembrane proteins. The protein contains 500 amino acids that form a large extracellular nidogen-like domain, a hydrophobic transmembrane domain, and a short cytoplasmic tail [76].

The increased TEM7 expression was established in aortic endothelial cells in mouse models [77]. Further, these authors have proven the TEM7 role in the capillary morphogenesis. Based on the reverse transcriptase-polymerase chain reaction (RT-PCR), TEM7 importance in the formation of functional microvascular proliferation and maintenance of neovascular endothelial cells in the fibrovascular membranes (FVMs) was shown [78]. These results suggest the crucial role of TEM7 expression in tumor-related blood capillaries formation. The modulation of TEM7 expression seems to be essential for blocking tumor capillaries to inhibit tumor growth [77].

TEM7 was first identified by St Croix et al. who found specific overexpression of this molecule in the endothelium of colorectal cancer [39]. Similarly, Rmali et al. [43] found the significantly higher expression of TEM7 in the tissue of colorectal cancer compared to healthy tissue $(77.5 \%$ versus $15 \%$ of positive tissue with TEM1 expression, resp.; $p<0.04)$. Consistent with these observations, significantly higher detection of TEM7 transcripts in colon carcinoma tissues than in the normal colon tissue (85\% versus $21 \%$ of TEM7 ISH+, resp.) was identified using in situ hybridization techniques performed on frozen tissues [79]. Considering these results, the TEM7 is suggested to be an attractive and perspective prognostic marker in CRC patients. The number of TEM7 transcripts was described to be higher in Dukes C compared to Dukes A colorectal tumor [43]. In this study, high level of TEM7 transcripts was associated with lymph nodes involvement and cancer progression. Knockdown of TEM7 resulted in a reduction of cell migration and invasion [80]. In gastric cell lines, downregulation of TEM7 by siRNA showed significant inhibition of gastric cell migration, approximately 50\% in SGC-7901 cell line and 60\% in AGS cell line. Moreover, TEM7 knockdown resulted in decreased cells invasion by $70 \%$, which led to reduced metastasis.

Except for overexpression of TEM7 in colorectal cancer, tests revealed high upregulation of TEM7 in the endothelium and perivascular cells of primary human cancers of the lung, pancreas, breast, brain, and osteogenic sarcoma $[8,76,81]$. Besides, soluble and secreted forms of TEM7 have been identified [76]. After that, it has been found that TEM7 via extracellular nidogen-like domain can interact with cortactin cytoplasmatic protein, and the binding region is minuscule (nine-amino-acid sequence). TEM7-cortactin interaction is supposed to be useful in the development of low weight molecules (peptides or analogs) that can be a promising tool for diagnosis and treatment of cancer by targeting tumor endothelium $[76,82]$.

Targeting angiogenesis is recognized as an effective way to promote cancer cell death in cancer patients' treatments. Therapy with anti-TEM7 seems to be a novel approach. Antibodies against TEM7 might interfere and block exclusively tumor blood vessels with elevated expression of TEM7. In comparison to therapies targeting, that is, VEGF pathway, the anti-TEM7 therapy might result in a reduction of side effects of antiangiogenic therapies, that is, hypertension, cardiotoxicity, or proteinuria [83]. TEM7 targeted in human SKOV3 ovarian carcinoma and MDA-MB-231 breast carcinoma cells showed perspective effects [79]. An anti-TEM7 antibody caused lysis of the cancer cells. Percentage of lysis in antiTEM7 targeted MDA-MB-231 cancer cells was higher than in SKOV3 cells (control assay) incubated with trastuzumab $(74.8 \pm 1.0 \%$ in MDA-MB-231 versus $61.8 \pm 7.2 \%$ in SKOV3). These results provide a new insight for antiangiogenic therapy of patients with some cancers.

Despite the in vivo experiments, the therapy targeting TEM7 calls for the clinical trials that could prove the immunomodulatory activity of anti-TEM7 antibodies. 
5.4. Tumor Endothelial Marker 8. Tumor endothelial marker 8 (TEM8, anthrax toxin receptor (ATR), and ANTXR1) is a type I transmembrane glycoprotein composed of 564 amino acids. TEM8 is homologous to capillary morphogenesis gene2 (CMG2, ANTXR2), also a type I transmembrane protein. TEM8 and CMG2 amino acids sequence is similar in $40 \%$ [84]. Both TEM8 and CMG2 proteins contain the extracellular domain, the von Willebrand factor A (vWF). The vWF domain homology is $60 \%$ between TEM8 and CMG2. Within the vWF domain of the TEM8/CMG2 proteins a conserved metal-ion-dependent adhesion site (MIDAS) motif is located. Two MIDAS conformations are recognized: closed (lowaffinity ligand binding state) and open (high-affinity ligand binding state) $[85,86]$. A metal ion-dependent adhesion site (MIDAS) is critical for binding the receptors. The vWF domain performs its hemostatic functions through the interaction with transmembrane receptors (from integrins superfamily) that make the bridges for cell-to-cell and cell-toextracellular matrix communications. Therefore, it has been identified as a unique endothelial marker in angiogenesis [87]. The interaction between TEM 8 and cellular matrix components suggests a potential role of TEM8 in adhesion and migration of cells [88]. Loss of TEM8 expression in mice resulted in embryonic and postnatal vascular and connective tissue defects, which leads to extensive hemorrhage and has had an increasing effect on deposition of extracellular matrix [89]. Therefore, the TEM8 involvement in the regulation of the balance between proliferative and fibrotic processes that occur during angiogenesis is proposed.

However, TEM8 and CMG2 bind proteins (collagen type $\mathrm{I}$, collagen type IV, and laminin) to extracellular matrix are different [78]. The TEM8 receptor is responsible for entry of anthrax toxin (produced by gram-positive bacterium Bacillus anthracis) into host cells and appears to regulate endothelial cell migration and tubule formation [85]. The CMG2 is involved in the homeostasis of the extracellular matrix [84]. These biochemical properties and the biologic behavior of TEM8 and CMG2 may be responsible for their apparent roles in regulating endothelial cell behavior during angiogenesis.

The presence of TEM8 on the cell surface is selectively controlled [87]. TEM8 is considered as a novel extracellular tumor marker among the other cell surface TEMs. TEM8 expression pattern is tumor-specific and has not been detected in physiologic angiogenesis [90, 91]. The TEM8 expression was found in various cancers, that is, breast and gallbladder [91-93]. Moreover, high TEM8 levels were associated with significantly shorter survival in breast cancer patients [91].

TEM8 is upregulated in tumor endothelium derived from human and mice colorectal tissue $[41,53,94]$. Overexpression of TEM8 was found in colon cancer tissue compared to normal colon tissue [95]. Additionally, TEM8 antibody showed the high TEM8 expression in microvessels of tumor tissue. Therefore, the TEM8 is recognized as a useful marker for identifying tumor-associated microvessels in CRC $[95,96]$. Results of the study of Raeisossadati et al. strongly confirmed the usefulness of TEM8 as a biomarker for the detection of CRC patients [97]. Authors found significantly higher circulating mRNA levels of TEM8 in the peripheral blood of
CRC patients (22/40) compared to healthy controls (9/40). It seems that TEM8 appears to be not only a promising marker of colorectal tumor presence but also the marker of tumor invasiveness and spread. Rmali et al. revealed an increased number of TEM8 transcript copies in colon cancer tissue in an advanced stage of disease (Dukes C: $73 \pm 0.5$ TEM8 transcript copies versus Dukes A: $32 \pm 0.5$ TEM8 transcript copies $p=0.01$ ) [95].

Since TEM8 expression is specific for tumor vasculature, antagonists of TEM8 might disrupt tumor angiogenesis and inhibit tumor progression $[39,98]$. Quan et al. [99] developed antibody (a 13-meric peptide, KYNDRLPLYISNP; QQM) able to bind with TEM8. The QQM peptide labeled with $18 \mathrm{~F}$ bound specifically to the extracellular domain of TEM8 in both the head-and-neck cancer and melanoma models. This property could serve as a new target in cancer imaging and therapy $[99,100]$. Chaudhary et al. [101] tested the L2 and L5, full IgG antibodies against TEM8 which was expressed in tumor xenografts including colon cancer (DLD1) in mice. The block of TEM8 expression resulted in tumor growth inhibition, similar to tumor growth impairment observed in the genetic ablation of TEM8. Fernando and Fletcher [94] evidenced that antibodies targeting TEM8 reduce tumor volume approximately of $53 \%$ and confirmed a tumor growth delay of $49 \%$ in the xenograft model of colorectal carcinoma compared to control mice. All of these data support the hypothesis that anti-TEM8 antibodies display potent antitumor activity. These antibodies are functionally involved in selective inhibition of angiogenesis and by that means indirectly block the tumor development [102].

The other molecules recognized as tumor endothelial markers and partially described are TEM2 and TEM4.

5.5. Tumor Endothelial Marker 2. Tumor endothelial marker 2 (TEM2, RASD family member 2): the protein functions as an activator of rapamycin 1 complex (mTOR1), which in turn is involved in multiple cellular functions, that is, regulating nutrient/energy/redox flow. Therefore, the activity of RASD/mTOR1 pathway controls the cell growth and proliferation by ensuring the resources and energy for protein synthesis [103]. The usefulness of TEM2 as an indicator for assessing the degree of tumor angiogenesis in colorectal cancer is limited. Although high expression of TEM2 is characteristic for colorectal cancer, it is also detected in the normal tissues (expression of TEM2: $45 \%$ in normal colon tissues versus $58.3 \%$ in tumor tissue; $p>0.05$ ). However, significantly high transcript copies of TEM2 were found in Dukes A compared to Dukes C colorectal cancer $[43,55]$. These patterns of TEM2 expression in cancer and healthy tissues reveal that TEM2 is not specific for tumor angiogenesis and makes the TEM2 doubtful marker in detection and targeting in tumor-related angiogenesis. Further investigations are needed to explore the TEM2 function in different tumors and to determine the possible role of TEM2 in tumor blood vessels formation.

5.6. Tumor Endothelial Marker 4. Tumor endothelial marker 4 (TEM4, ARHGEF17) is a Rho-specific guanine nucleotide 
exchange factor. It contains the $\mathrm{DH}$ and $\mathrm{PH}$ domains, extended $\mathrm{N}$-terminal sequences with no identifiable domains or motifs, and a C-terminal domain [104]. TEM4 is localized in the subcellular compartment. The biological role of TEM4 is associated with regulating activation of Rho GTPases, various members of the cadherin-catenin complex, and several cytoskeleton proteins $[105,106]$. TEM4 upregulation was identified in the endothelial cells during tumor angiogenesis of colorectal cancer patients [39]. Consistent with the TEM4 central role in controlling activation of RhoC in endothelial cells, the organization of actin cytoskeleton, cell-substrate adhesion, and cellular migration, it is suggested that TEM4 is critical for blood vessel formation during angiogenesis [105].

\section{Conclusion}

The correlation between predominant TEMs expressions in the endothelium strongly suggests a significant involvement of TEMs in tumor blood vessels formation. The development of biomarkers-based diagnostic tests is still a great challenge in cancer diagnosis and therapy. Overexpression of TEM1, TEM7, and TEM8 in colorectal tumor tissue compared to healthy tissue suggests their role in pathological angiogenesis. TEMs specifically expressed in tumor tissue provide potential novel targets to develop diagnostic and therapeutic molecules. Anti-TEMs antibodies provide a promising new tool for selective identification of pathological vessels. It appears that block of TEMs expression is a crucial therapeutic pathway in angiogenesis-dependent diseases, including colorectal cancer.

However, many questions about TEMs remain undiscovered and will need to be addressed in future molecular and clinical studies. Further dissection of the TEMs pathway should provide much insight into the molecular mechanism of cancer angiogenesis and its regulation.

\section{Competing Interests}

The author declares that there is no conflict of interest regarding the publication of this paper.

\section{Acknowledgments}

This study was supported by the Medical University of Lublin (Grant no. MNmb 230).

\section{References}

[1] J. Ferlay, I. Soerjomataram, M. Ervik et al., GLOBOCAN 2012 v1.0, Cancer Incidence and Mortality Worldwide: IARC CancerBase No. 11, International Agency for Research on Cancer, Lyon, France, 2013, http://globocan.iarc.fr.

[2] L. Pietrzyk, A. Torres, R. Maciejewski, and K. Torres, "Obesity and obese-related chronic low-grade inflammation in promotion of colorectal cancer development," Asian Pacific Journal of Cancer Prevention, vol. 16, no. 10, pp. 4161-4168, 2015.

[3] D. M. Parkin, F. Bray, J. Ferlay, and P. Pisani, "Global cancer statistics, 2002," CA: A Cancer Journal for Clinicians, vol. 55, no. 2, pp. 74-108, 2005.
[4] G. A. Ganepola, J. Nizin, J. R. Rutledge, and D. H. Chang, "Use of blood-based biomarkers for early diagnosis and surveillance of colorectal cancer," World Journal of Gastrointestinal Oncology, vol. 6, no. 4, pp. 83-97, 2014.

[5] National Cancer Institute, "SEER Stat Fact Sheets: Colon and Rectum Cancer," http://seer.cancer.gov/statfacts/html/colorect .html.

[6] M. Gonzalez-Pons and M. Cruz-Correa, "Colorectal cancer biomarkers: where are we now?" BioMed Research International, vol. 2015, Article ID 149014, 14 pages, 2015.

[7] P. Beaudry, J. Force, G. N. Naumov et al., "Differential effects of vascular endothelial growth factor receptor-2 inhibitor ZD6474 on circulating endothelial progenitors and mature circulating endothelial cells: implications for use as a surrogate marker of antiangiogenic activity," Clinical Cancer Research, vol. 11, no. 9, pp. 3514-3522, 2005.

[8] R. Mehran, M. Nilsson, M. Khajavi et al., "Tumor endothelial markers define novel subsets of cancer-specific circulating endothelial cells associated with antitumor efficacy," Cancer Research, vol. 74, no. 10, pp. 2731-2741, 2014.

[9] G. Lech, R. Slotwinski, and I. W. Krasnodebski, "The role of tumor markers and biomarkers in colorectal cancer," Neoplasma, vol. 61, no. 1, pp. 1-8, 2014.

[10] J. Folkman, "History of angiogenesis," in Angiogenesis-An Integrative Approach from Science to Medicine, W. D. Figg and J. Folkman, Eds., pp. 1-14, Springer, New York, NY, USA, 2008.

[11] G. N. Naumov, L. A. Akslen, and J. Folkman, "Role of angiogenesis in human tumor dormancy: animal models of the angiogenic switch," Cell Cycle, vol. 5, no. 16, pp. 1779-1787, 2006.

[12] J. Folkman, "Role of angiogenesis in tumor growth and metastasis," Seminars in Oncology, vol. 29, no. 6, supplement 16, pp. 15-18, 2002.

[13] J. Folkman and D. Hanahan, "Switch to the angiogenic phenotype during tumorigenesis," Princess Takamatsu Symposia, vol. 22, pp. 339-347, 1991.

[14] T. F. McGuire, G. B. Sajithlal, J. Lu, R. D. Nicholls, and E. V. Prochownik, "In vivo evolution of tumor-derived endothelial cells," PLoS ONE, vol. 7, no. 5, Article ID e37138, 2012.

[15] S. Koch and L. Claesson-Welsh, "Signal transduction by vascular endothelial growth factor receptors," Cold Spring Harbor Perspectives in Medicine, vol. 2, no. 7, Article ID a006502, 2012.

[16] H. Hashizume, P. Baluk, S. Morikawa et al., "Openings between defective endothelial cells explain tumor vessel leakiness," The American Journal of Pathology, vol. 156, no. 4, pp. 1363-1380, 2000.

[17] C. Schrimpf, O. E. Teebken, M. Wilhelmi, and J. S. Duffield, "The role of pericyte detachment in vascular rarefaction," Journal of Vascular Research, vol. 51, no. 4, pp. 247-258, 2014.

[18] A. M. Dvorak, S. Kohn, E. S. Morgan, P. Fox, J. A. Nagy, and H. F. Dvorak, "The vesiculo-vacuolar organelle (VVO): a distinct endothelial cell structure that provides a transcellular pathway for macromolecular extravasation," Journal of Leukocyte Biology, vol. 59, no. 1, pp. 100-115, 1996.

[19] J. A. Nagy, L. Benjamin, H. Zeng, A. M. Dvorak, and H. F. Dvorak, "Vascular permeability, vascular hyperpermeability and angiogenesis," Angiogenesis, vol. 11, no. 2, pp. 109-119, 2008.

[20] D. M. McDonald and P. L. Choyke, "Imaging of angiogenesis: from microscope to clinic," Nature Medicine, vol. 9, no. 6, pp. 713-725, 2003.

[21] R. Kalluri, "Basement membranes: structure, assembly and role in tumour angiogenesis," Nature Reviews Cancer, vol. 3, no. 6, pp. 422-433, 2003. 
[22] P. Baluk, H. Hashizume, and D. M. McDonald, "Cellular abnormalities of blood vessels as targets in cancer," Current Opinion in Genetics and Development, vol. 15, no. 1, pp. 102-111, 2005.

[23] D. M. McDonald and P. Baluk, "Significance of blood vessel leakiness in cancer," Cancer Research, vol. 62, no. 18, pp. 5381$5385,2002$.

[24] R. K. Jain, "Normalization of tumor vasculature: an emerging concept in antiangiogenic therapy," Science, vol. 307, no. 5706, pp. 58-62, 2005.

[25] A. C. Dudley, "Tumor endothelial cells," Cold Spring Harbor Perspectives in Medicine, vol. 2, no. 3, Article ID a006536, 2012.

[26] G. Bergers and D. Hanahan, "Modes of resistance to antiangiogenic therapy," Nature Reviews Cancer, vol. 8, no. 8, pp. 592-603, 2008.

[27] A. Armulik, G. Genové, and C. Betsholtz, "Pericytes: developmental, physiological, and pathological perspectives, problems, and promises," Developmental Cell, vol. 21, no. 2, pp. 193-215, 2011.

[28] A. Armulik, A. Abramsson, and C. Betsholtz, "Endothelial/pericyte interactions," Circulation Research, vol. 97, no. 6, pp. 512-523, 2005.

[29] D. Gao, D. J. Nolan, A. S. Mellick, K. Bambino, K. McDonnell, and V. Mittal, "Endothelial progenitor cells control the angiogenic switch in mouse lung metastasis," Science, vol. 319, no. 5860, pp. 195-198, 2008.

[30] D. J. Nolan, A. Ciarrocchi, A. S. Mellick et al., "Bone marrowderived endothelial progenitor cells are a major determinant of nascent tumor neovascularization," Genes and Development, vol. 21, no. 12, pp. 1546-1558, 2007.

[31] T. Asahara, T. Murohara, A. Sullivan et al., "Isolation of putative progenitor endothelial cells for angiogenesis," Science, vol. 275, no. 5302, pp. 964-967, 1997.

[32] D. A. Ingram, L. E. Mead, H. Tanaka et al., "Identification of a novel hierarchy of endothelial progenitor cells using human peripheral and umbilical cord blood," Blood, vol. 104, no. 9, pp. 2752-2760, 2004.

[33] M. C. Yoder, "Human endothelial progenitor cells," Cold Spring Harbor Perspectives in Medicine, vol. 2, no. 7, Article ID a006692, 2012.

[34] N. Quirici, D. Soligo, L. Caneva, F. Servida, P. Bossolasco, and G. L. Deliliers, "Differentiation and expansion of endothelial cells from human bone marrow $\mathrm{CD}_{133^{+}}$cells," British Journal of Haematology, vol. 115, no. 1, pp. 186-194, 2001.

[35] M. Hristov, W. Erl, and P. C. Weber, "Endothelial progenitor cells: mobilization, differentiation, and homing," Arteriosclerosis, Thrombosis, and Vascular Biology, vol. 23, no. 7, pp. 11851189, 2003.

[36] S. Kaushal, G. E. Amiel, K. J. Guleserian et al., "Functional small-diameter neovessels created using endothelial progenitor cells expanded ex vivo," Nature Medicine, vol. 7, no. 9, pp. 10351040, 2001.

[37] M. C. Deregibus, V. Cantaluppi, R. Calogero et al., "Endothelial progenitor cell-derived microvesicles activate an angiogenic program in endothelial cells by a horizontal transfer of mRNA," Blood, vol. 110, no. 7, pp. 2440-2448, 2007.

[38] D. G. Duda, K. S. Cohen, E. di Tomaso et al., "Differential CD146 expression on circulating versus tissue endothelial cells in rectal cancer patients: implications for circulating endothelial and progenitor cells as biomarkers for antiangiogenic therapy," Journal of Clinical Oncology, vol. 24, no. 9, pp. 1449-1453, 2006.
[39] B. St Croix, C. Rago, V. Velculescu et al., "Genes expressed in human tumor endothelium," Science, vol. 289, no. 5482, pp. 1197-1202, 2000.

[40] S. Seaman, J. Stevens, M. Y. Yang, D. Logsdon, C. Graff-Cherry, and B. St Croix, "Genes that distinguish physiological and pathological angiogenesis," Cancer Cell, vol. 11, no. 6, pp. 539554, 2007.

[41] E. B. Carson-Walter, D. N. Watkins, A. Nanda, B. Vogelstein, K. W. Kinzler, and B. St Croix, "Cell surface tumor endothelial markers are conserved in mice and humans," Cancer Research, vol. 61, no. 18, pp. 6649-6655, 2001.

[42] M. Mesri, C. Birse, J. Heidbrink et al., "Identification and characterization of angiogenesis targets through proteomic profiling of endothelial cells in human cancer tissues," PLoS ONE, vol. 8, no. 11, Article ID e78885, 2013.

[43] K. A. Rmali, M. C. A. Puntis, and W. G. Jiang, "Prognostic values of tumor endothelial markers in patients with colorectal cancer," World Journal of Gastroenterology, vol. 11, no. 9, pp. 1283-1286, 2005.

[44] K. Hida, Y. Hida, and M. Shindoh, "Understanding tumor endothelial cell abnormalities to develop ideal anti-angiogenic therapies," Cancer Science, vol. 29, no. 3, pp. 459-466, 2008.

[45] A. Nanda and B. St Croix, "Tumor endothelial markers: new targets for cancer therapy," Current Opinion in Oncology, vol. 16, no. 1, pp. 44-49, 2004.

[46] R. G. Bagley, "Endosialin: from vascular target to biomarker for human sarcomas," Biomarkers in Medicine, vol. 3, no. 5, pp. 589604, 2009.

[47] Z.-Y. Zhang, H. Zhang, G. Adell, and X.-F. Sun, "Endosialin expression in relation to clinicopathological and biological variables in rectal cancers with a Swedish clinical trial of preoperative radiotherapy," BMC Cancer, vol. 11, article 89, 2011.

[48] R. Opavsky, P. Haviernik, D. Jurkovicova et al., "Molecular characterization of the mouse Tem1/endosialin gene regulated by cell density in vitro and expressed in normal tissues in vivo," The Journal of Biological Chemistry, vol. 276, no. 42, pp. 38795$38807,2001$.

[49] A. Ohradanova, K. Gradin, M. Barathova et al., "Hypoxia upregulates expression of human endosialin gene via hypoxiainducible factor 2," British Journal of Cancer, vol. 99, no. 8, pp. 1348-1356, 2008.

[50] D. Liao and R. S. Johnson, "Hypoxia: a key regulator of angiogenesis in cancer," Cancer and Metastasis Reviews, vol. 26, no. 2, pp. 281-290, 2007.

[51] G. Elvert, A. Kappel, R. Heidenreich et al., "Cooperative interaction of hypoxia inducible factor- $2 \alpha$ and Ets-1 in the transcriptional activation of vascular endothelial growth factor receptor-2 (Flk-1)," The Journal of Biological Chemistry, vol. 278, no. 9, pp. 7520-7530, 2003.

[52] A. Le Bras, F. Lionneton, V. Mattot et al., "HIF-2alpha specifically activates the VE-cadherin promoter independently of hypoxia and in synergy with Ets-1 through two essential ETSbinding sites," Oncogene, vol. 26, no. 53, pp. 7480-7489, 2007.

[53] A. Nanda, B. Karim, Z. Peng et al., "Tumor endothelial marker 1 (Teml) functions in the growth and progression of abdominal tumors," Proceedings of the National Academy of Sciences of the United States of America, vol. 103, no. 9, pp. 3351-3356, 2006.

[54] B. Tomkowicz, K. Rybinski, B. Foley et al., "Interaction of endosialin/TEM1 with extracellular matrix proteins mediates cell adhesion and migration," Proceedings of the National Academy of Sciences of the United States of America, vol. 104, no. 46, pp. 17965-17970, 2007. 
[55] G. Davies, G. H. Cunnick, R. E. Mansel, M. D. Mason, and W. G. Jiang, "Levels of expression of endothelial markers specific to tumour-associated endothelial cells and their correlation with prognosis in patients with breast cancer," Clinical and Experimental Metastasis, vol. 21, no. 1, pp. 31-37, 2004.

[56] H. Dolznig, N. Schweifer, C. Puri et al., "Characterization of cancer stroma markers: In silico analysis of an mRNA expression database for fibroblast activation protein and endosialin," Cancer Immunity, vol. 5, article 10, 2005.

[57] J. Brady, J. Neal, N. Sadakar, and P. Gasque, "Human endosialin (tumor endothelial marker 1) is abundantly expressed in highly malignant and invasive brain tumors," Journal of Neuropathology and Experimental Neurology, vol. 63, no. 12, pp. 1274-1283, 2004.

[58] M. A. Huber, N. Kraut, N. Schweifer et al., "Expression of stromal cell markers in distinct compartments of human skin cancers," Journal of Cutaneous Pathology, vol. 33, no. 2, pp. 145155, 2006.

[59] J. R. MacFadyen, O. Haworth, D. Roberston et al., "Endosialin (TEM1, CD248) is a marker of stromal fibroblasts and is not selectively expressed on tumour endothelium," FEBS Letters, vol. 579, no. 12, pp. 2569-2575, 2005.

[60] D. Virgintino, F. Girolamo, M. Errede et al., "An intimate interplay between precocious, migrating pericytes and endothelial cells governs human fetal brain angiogenesis," Angiogenesis, vol. 10, no. 1, pp. 35-45, 2007.

[61] D. von Tell, A. Armulik, and C. Betsholtz, "Pericytes and vascular stability," Experimental Cell Research, vol. 312, no. 5, pp. 623-629, 2006.

[62] A.-M. Chacko, C. Li, M. Nayak et al., "Development of 124I immuno-pet targeting tumor vascular TEM1/endosialin," Journal of Nuclear Medicine, vol. 55, no. 3, pp. 500-507, 2014.

[63] S. E. Lange, A. Zheleznyak, M. Studer, D. J. O’Shannessy, S. E. Lapi, and B. A. Van Tine, "Development of 89Zr-Ontuxizumab for in vivo TEM-1/endosialin PET applications," Oncotarget, vol. 7, no. 11, pp. 13082-13092, 2016.

[64] J. G. Facciponte, S. Ugel, F. De Sanctis et al., "Tumor endothelial marker 1-specific DNA vaccination targets tumor vasculature," The Journal of Clinical Investigation, vol. 124, no. 4, pp. 1497-1511, 2014.

[65] L. A. Diaz, C. M. Coughlin, S. C. Weil et al., "A first-inhuman phase i study of MORAb-004, a monoclonal antibody to endosialin in patients with advanced solid tumors," Clinical Cancer Research, vol. 21, no. 6, pp. 1281-1288, 2015.

[66] M. Maia, A. DeVriese, T. Janssens et al., "CD248 facilitates tumor growth via its cytoplasmic domain,” BMC Cancer, vol. 11, article 162, 2011.

[67] I. Wierzbicka-Patynowski and J. E. Schwarzbauer, "The ins and outs of fibronectin matrix assembly," Journal of Cell Science, vol. 116, no. 16, pp. 3269-3276, 2003.

[68] M. Vallon, F. Rohde, K.-P. Janssen, and M. Essler, "Tumor endothelial marker 5 expression in endothelial cells during capillary morphogenesis is induced by the small GTPase Rac and mediates contact inhibition of cell proliferation," Experimental Cell Research, vol. 316, no. 3, pp. 412-421, 2010.

[69] Y. Yamamoto, K. Irie, M. Asada, A. Mino, K. Mandai, and Y. Takai, "Direct binding of the human homologue of the Drosophila disc large tumor suppressor gene to seven-pass transmembrane proteins, tumor endothelial marker 5 (TEM5), and a novel TEM5-like protein," Oncogene, vol. 23, no. 22, pp. 3889-3897, 2004.
[70] E. Ruoslahti, "RGD and other recognition sequences for integrins," Annual Review of Cell and Developmental Biology, vol. 12, pp. 697-715, 1996.

[71] T. Ishidate, A. Matsumine, K. Toyoshima, and T. Akiyama, "The APC-hDLG complex negatively regulates cell cycle progression from the G0/G1 to S phase," Oncogene, vol. 19, no. 3, pp. 365-372, 2000.

[72] E. Posokhova, A. Shukla, S. Seaman et al., "GPR124 functions as a WNT7-specific coactivator of canonical $\beta$-catenin signaling," Cell Reports, vol. 10, no. 2, pp. 123-130, 2015.

[73] C. P. Neal, A. M. Fry, C. Moreman et al., "Overexpression of the Nek2 kinase in colorectal cancer correlates with beta-catenin relocalization and shortened cancer-specific survival," Journal of Surgical Oncology, vol. 110, no. 7, pp. 828-838, 2014.

[74] Y. Wang, S.-G. Cho, X. Wu, S. Siwko, and M. Liu, "G-protein coupled receptor 124 (GPR124) in endothelial cells regulates vascular endothelial growth factor (VEGF)-induced tumor angiogenesis," Current Molecular Medicine, vol. 14, no. 4, pp. 543-554, 2014.

[75] Y. Gao, X. Fan, W. Li, W. Ping, Y. Deng, and X. Fu, "miR-138$5 p$ reverses gefitinib resistance in non-small cell lung cancer cells via negatively regulating G protein-coupled receptor 124 ," Biochemical and Biophysical Research Communications, vol. 446, no. 1, pp. 179-186, 2014.

[76] A. Nanda, P. Buckhaults, S. Seaman et al., "Identification of a binding partner for the endothelial cell surface proteins TEM7 and TEM7R," Cancer Research, vol. 64, no. 23, pp. 8507-8511, 2004.

[77] X.-Q. Wang, N. Sheibani, and J. C. Watson, "Modulation of tumor endothelial cell marker 7 expression during endothelial cell capillary morphogenesis," Microvascular Research, vol. 70, no. 3, pp. 189-197, 2005.

[78] Y. Yamaji, S. Yoshida, K. Ishikawa et al., “TEM7 (PLXDC1) in neovascular endothelial cells of fibrovascular membranes from patients with proliferative diabetic retinopathy," Investigative Ophthalmology and Visual Science, vol. 49, no. 7, pp. 3151-3157, 2008.

[79] R. G. Bagley, C. Rouleau, W. Weber et al., "Tumor endothelial marker 7 (TEM-7): a novel target for antiangiogenic therapy," Microvascular Research, vol. 82, no. 3, pp. 253-262, 2011.

[80] Z. Z. Zhang, R. Hua, J. F. Zhang et al., “TEM7 (PLXDC1), a key prognostic predictor for resectable gastric cancer, promotes cancer cell migration and invasion," American Journal of Cancer Research, vol. 5, pp. 772-781, 2015.

[81] B. Fuchs, E. Mahlum, C. Halder et al., "High expression of tumor endothelial marker 7 is associated with metastasis and poor survival of patients with osteogenic sarcoma," Gene, vol. 399, no. 2, pp. 137-143, 2007.

[82] A. Ries, W. Göhring, J. W. Fox, R. Timpl, and T. Sasaki, "Recombinant domains of mouse nidogen-1 and their binding to basement membrane proteins and monoclonal antibodies," European Journal of Biochemistry, vol. 268, no. 19, pp. 5119-5128, 2001.

[83] K. M. Cook and W. D. Figg, "Angiogenesis inhibitors: current strategies and future prospects," CA: Cancer Journal for Clinicians, vol. 60, no. 4, pp. 222-243, 2010.

[84] H. M. Scobie, G. J. A. Rainey, K. A. Bradley, and J. A. T. Young, "Human capillary morphogenesis protein 2 functions as an anthrax toxin receptor," Proceedings of the National Academy of Sciences of the United States of America, vol. 100, no. 9, pp. 5170$5174,2003$. 
[85] S. Fu, X. Tong, C. Cai et al., "The structure of tumor endothelial marker 8 (TEM8) extracellular domain and implications for its receptor function for recognizing anthrax toxin," PLOS ONE, vol. 5, no. 6, Article ID el1203, 2010.

[86] D. B. Lacy, D. J. Wigelsworth, H. M. Scobie, J. A. T. Young, and R. J. Collier, "Crystal structure of the von Willebrand factor A domain of human capillary morphogenesis protein 2: an anthrax toxin receptor," Proceedings of the National Academy of Sciences of the United States of America, vol. 101, no. 17, pp. 63676372, 2004.

[87] J. D. Hood and D. A. Cheresh, "Role of integrins in cell invasion and migration," Nature Reviews Cancer, vol. 2, no. 2, pp. 91-100, 2002.

[88] M. Y. Yang, A. Chaudhary, S. Seaman et al., "The cell surface structure of tumor endothelial marker 8 (TEM8) is regulated by the actin cytoskeleton," Biochimica et Biophysica Acta (BBA)Molecular Cell Research, vol. 1813, no. 1, pp. 39-49, 2011.

[89] T. Y. Besschetnova, T. Ichimura, N. Katebi, B. St Croix, J. V. Bonventre, and B. R. Olsen, "Regulatory mechanisms of anthrax toxin receptor 1-dependent vascular and connective tissue homeostasis," Matrix Biology, vol. 42, pp. 56-73, 2015.

[90] S. E. Bell, A. Mavila, R. Salazar et al., "Differential gene expression during capillary morphogenesis in $3 \mathrm{D}$ collagen matrices: regulated expression of genes involved in basement membrane matrix assembly, cell cycle progression, cellular differentiation and G-protein signaling," Journal of Cell Science, vol. 114, no. 15, pp. 2755-2773, 2001.

[91] K. A. Rmali, M. C. A. Puntis, and W. G. Jiang, "TEM-8 and tubule formation in endothelial cells, its potential role of its vW/TM domains," Biochemical and Biophysical Research Communications, vol. 334, no. 1, pp. 231-238, 2005.

[92] G. Davies, K. A. Rmali, G. Watkins, R. E. Mansel, M. D. Mason, and W. G. Jiang, "Elevated levels of tumour endothelial marker-8 in human breast cancer and its clinical significance," International Journal of Oncology, vol. 29, no. 5, pp. 1311-1317, 2006.

[93] S. K. Maurya, M. Tewari, M. Kumar, M. K. Thakur, and H. S. Shukla, "Expression pattern of tumor endothelial marker 8 protein in gallbladder carcinomas," Asian Pacific Journal of Cancer Prevention, vol. 12, no. 2, pp. 507-512, 2011.

[94] S. Fernando and B. S. Fletcher, "Targeting tumor endothelial marker 8 in the tumor vasculature of colorectal carcinomas in mice," Cancer Research, vol. 69, no. 12, pp. 5126-5132, 2009.

[95] K. A. Rmali, G. Watkins, G. Harrison, C. Parr, M. C. A. Puntis, and W. G. Jiang, "Tumour endothelial marker 8 (TEM8 ) in human colon cancer and its association with tumour progression," European Journal of Surgical Oncology, vol. 30, no. 9, pp. 948-953, 2004.

[96] Y. Wang, Z. Luo, K. Liu, J. Wang, and Y. Duan, "In situ targeting TEM8 via immune response and polypeptide recognition by wavelength-modulated surface plasmon resonance biosensor," Scientific Reports, vol. 6, Article ID 20006, 2016.

[97] R. Raeisossadati, M. Farshchian, A. Ganji et al., "Quantitative analysis of TEM-8 and CEA tumor markers indicating free tumor cells in the peripheral blood of colorectal cancer patients," International Journal of Colorectal Disease, vol. 26, no. 10, pp. 1265-1270, 2011.

[98] D. Herrmann, A. Ferrer-Vaquer, C. Lahsnig, N. Firnberg, A. Leibbrandt, and A. Neubüser, "Expression and regulation of ANTXR1 in the chick embryo," Developmental Dynamics, vol. 239, no. 2, pp. 680-687, 2010.
[99] Q. Quan, M. Yang, H. Gao et al., "Imaging tumor endothelial marker 8 using an ${ }^{18}$ F-labeled peptide," European Journal of Nuclear Medicine and Molecular Imaging, vol. 38, no. 10, pp. 1806-1815, 2011.

[100] S. H. Leppla, J. B. Robbins, R. Schneerson, and J. Shiloach, "Development of an improved vaccine for anthrax," Journal of Clinical Investigation, vol. 110, no. 2, pp. 141-144, 2002.

[101] A. Chaudhary, M. B. Hilton, S. Seaman et al., “TEM8/ANTXR1 blockade inhibits pathological angiogenesis and potentiates tumoricidal responses against multiple cancer types," Cancer Cell, vol. 21, no. 2, pp. 212-226, 2012.

[102] A. Chaudhary and B. St Croix, "Selective blockade of tumor angiogenesis," Cell Cycle, vol. 11, no. 12, pp. 2253-2259, 2012.

[103] Z. Ye, A. Q. O. Al-Aidaroos, J. E. Park et al., "PRL-3 activates mTORCl in cancer progression," Scientific Reports, vol. 5, article 17046, 2015.

[104] N. Mitin, K. L. Rossman, and C. J. Der, "Identification of a novel actin-binding domain within the Rho guanine nucleotide exchange factor TEM4," PLoS ONE, vol. 7, article e41876, 2012.

[105] N. Mitin, K. L. Rossman, R. Currin et al., “The RhoGEF TEM4 regulates endothelial cell migration by suppressing actomyosin contractility," PLoS ONE, vol. 8, article e66260, 2013.

[106] S. P. Ngok, R. Geyer, A. Kourtidis et al., "TEM4 is a junctional Rho GEF required for cell-cell adhesion, monolayer integrity and barrier function," Journal of Cell Science, vol. 126, no. 15, pp. 3271-3277, 2013. 


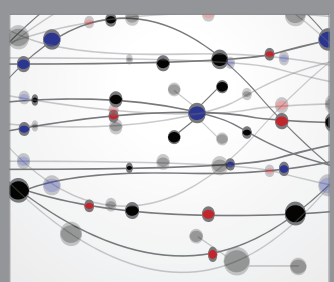

The Scientific World Journal
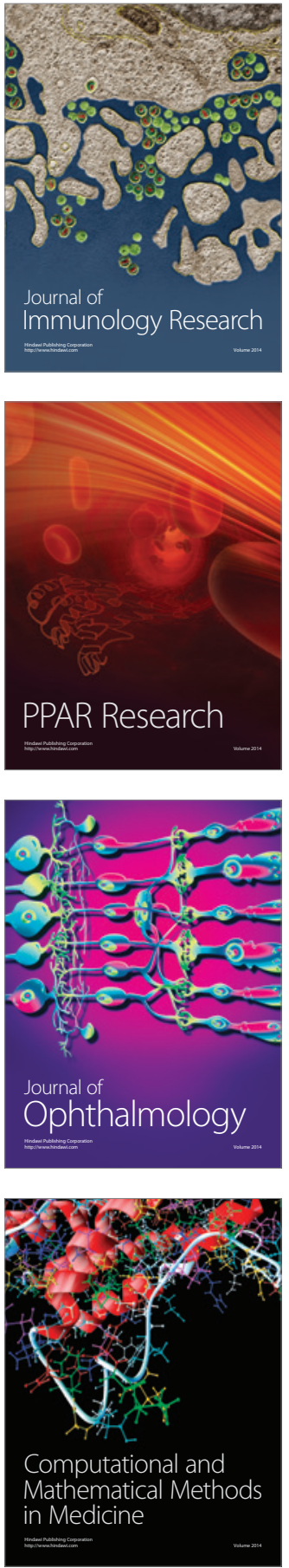

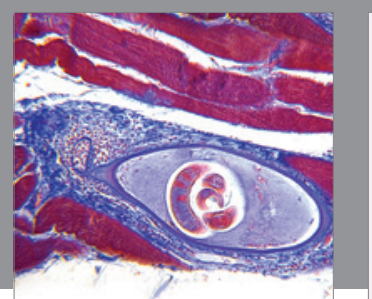

Gastroenterology Research and Practice

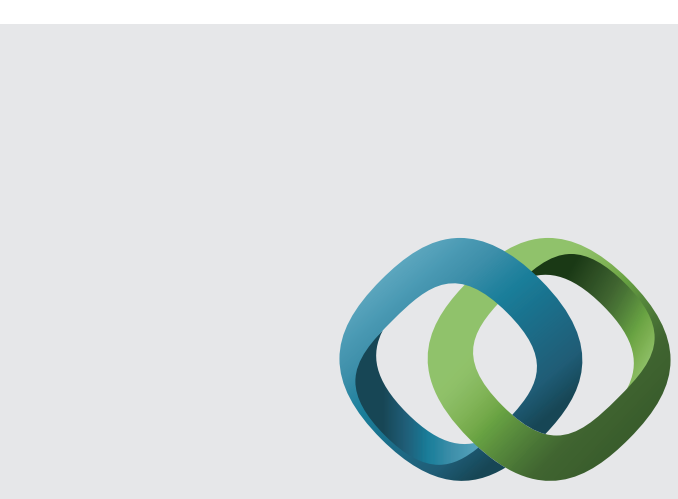

\section{Hindawi}

Submit your manuscripts at

http://www.hindawi.com
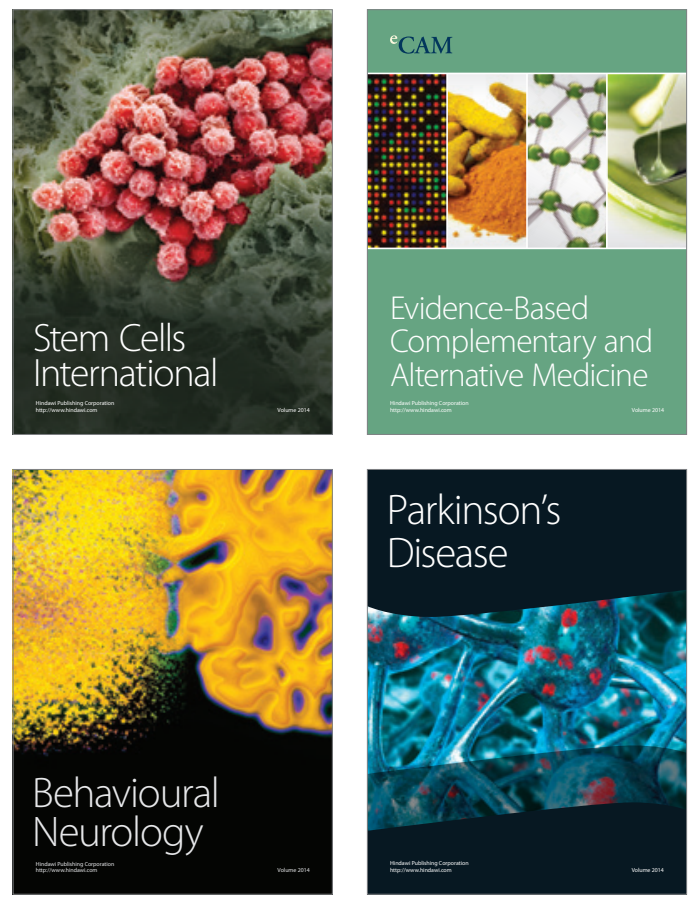
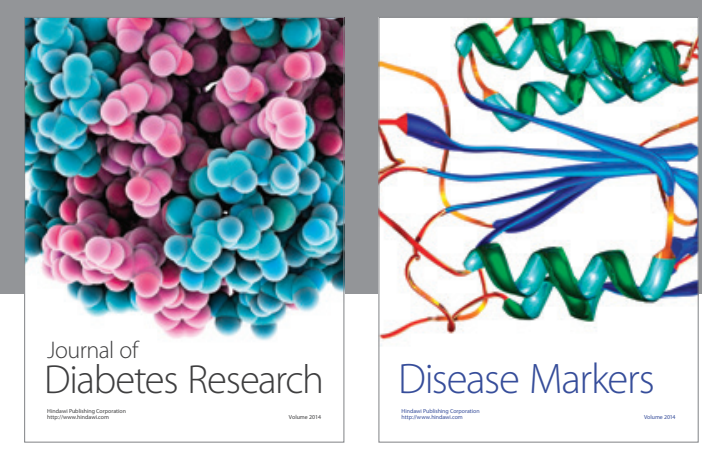

Disease Markers
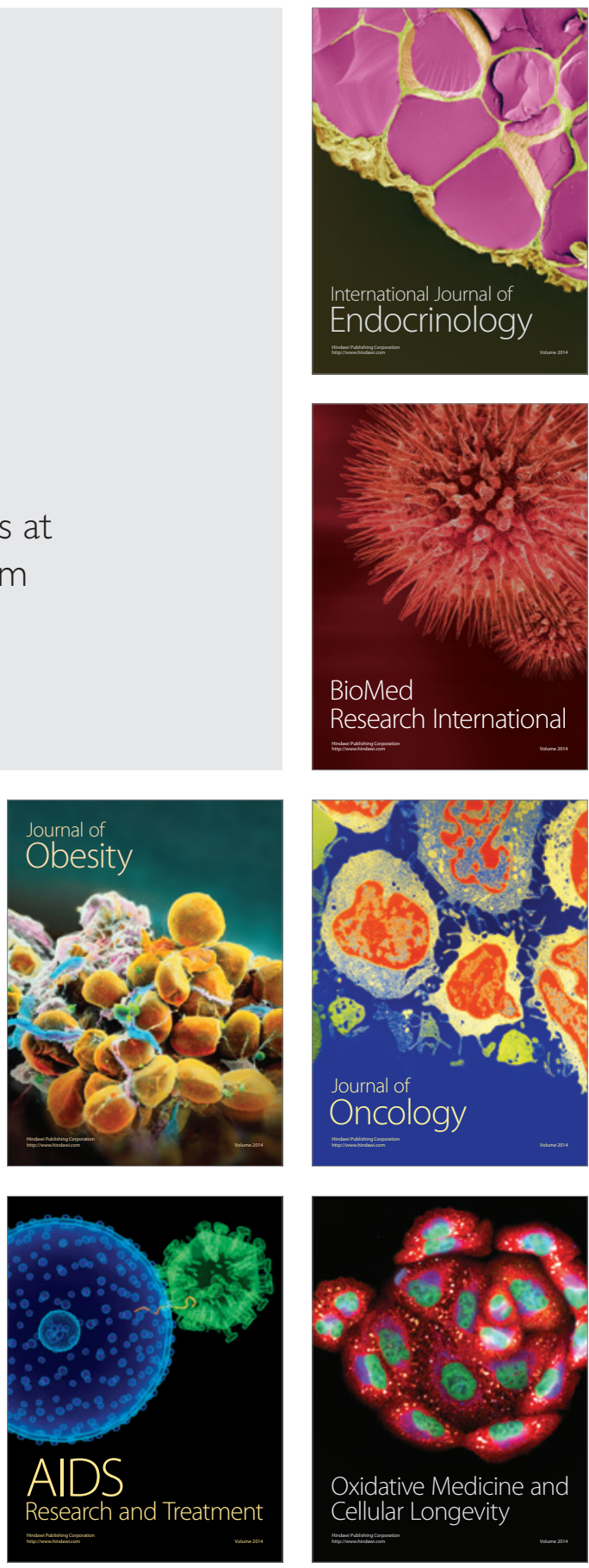